\title{
L'APPLICATION DU DROIT ELECTORAL PAR LE JUGE CONSTITUTIONNEL GABONAIS ET CONGOLAIS : UN RENDEZ-VOUS MANQUÉ POUR L'ÉTAT DE DROIT EN AFRIQUE CENTRALE
}

\author{
Valéry Iragi Ntwali ${ }^{\times}$
}

\section{Résumé}

Cette étude essaye de décrire le rôle partisan et dangereux que joue le juge électoral dans le règlement des litiges relatifs au contentieux électoral de l'élection présidentielle et législative en Afrique francophone et particulièrement, au Gabon et en RD Congo. Le regard a été fixé sur le contentieux de l'élection présidentielle et législative de 2016 au Gabon et celles de 2011 et 2018 en RD Congo. L'économie générale du droit électoral dans les deux pays étudiés, reconnaît la compétence de la Cour constitutionnelle en matière de contentieux de l'élection présidentielle et législative nationale. Mais le travail de ces Cours constitutionnelles en matière des contentieux électoraux de l'élection présidentielle et législative, dénote véritablement un rendez-vous manqué dans un État de droit, le constat restant mitigé par rapport à l'apport du juge électoral dans la légitimation du processus électoral et des résultats finaux. Ce qui fait que les arrêts de deux Cours évoquées, n'ont fait qu'amplifier les violences postélectorales au côté des discours politiques animés par des contestations et la manipulation populaire qui ont donné lieu, à des violations massives des droits de l'homme à travers une répression sanglante des manifestants, et à l'entérinement des push électoraux au Gabon et en RDC.

Mots Clés : application, droit électoral, juge constitutionnel

\section{Abstract}

\footnotetext{
×Valéry Iragi Ntwali est doctorant en Science politique à l'Université Paris-Est Créteil (Paris-12). Avocat au Barreau du Sud-Kivu, enseignant-Chercheur d'université en droit et science politique. Il est expert en relations internationales, spécialiste des questions de développement et de coopération Europe-Afrique. Il est également spécialiste des droits de l'homme et de droit Public. Il Possède un double Master en relations internationales et études du développement Europe-Afrique (Université de Szeged/Hongrie et Université d'Alexandrie/Egypte), un diplôme d'étude supérieure spécialisée en Droits de l'homme (DESS-CERDHO/UCB) et une Licence en Droit public $(\mathrm{Bac}+5 / \mathrm{UCB})$. Chercheur au Laboratoire Interdisciplinaire d'étude du Politique Hannah Arendt (LIPHAPE) des Universités Paris-Est Créteil et Gustave EIFFEL et au Centre Congolais de Justice Transitionnelle et de Sécurité Humaine (CCJT-SH).
} 
This study attempts to describe the partisan and dangerous role that the electoral judge plays in order to regulate the litigations related to the electoral disputes of the presidential and legislative elections in French-speaking Africa and particularly in Gabon and DR Congo. Attention was paid to the litigation of the presidential and legislative elections of 2016 in Gabon and those of 2011 and 2018 in DR Congo. The general economy of the electoral law in the two aforementioned countries, recognizes the competence of the Constitutional Court in the litigations related to presidential and national legislative elections. However, the work of the constitutional courts presented above, really denotes an appointment missed in a state of law, the finding remains mixed with respect to the contribution of the electoral judge in the legitimization of the electoral process and final results. As a result, the judgments of two Courts evoked only amplified post-election violence alongside political speeches fuelled by protests and popular manipulation, which caused massive violations of human rights through a bloody crackdown on protesters, and the endorsement of electoral push in Gabon and the DRC.

Keys-words : application, electoral law, constitutional judge

\section{Introduction}

Le Centre Carter soutient «qu'un consensus existe désormais au plan international sur le fait que des élections démocratiques honnêtes sont indispensables pour affirmer la légitimité des gouvernements $»^{1}$. Notons que le vent de démocratisation qui devait emporter les régimes autoritaires de l'Europe de l'Est devait embraser les régimes autoritaires africains sous l'impulsion d'autres facteurs, notamment internationaux, économiques et financiers ${ }^{2}$. Il est vrai qu'aujourd'hui, le recours aux élections est consacré par les textes constitutionnels, et la communauté internationale donne un privilège au régime démocratique, mais en Afrique francophone, ces élections rencontrent un grand nombre des problèmes, qui suscitent une appréhension de la part des chercheurs en droit et en sciences sociales ${ }^{3}$.

Pour Jean du BOIS de GAUDUSSON « les difficultés semblent parfois empirer si l'on en juge par la gravité des crises liées à l'organisation de récents scrutins (Côte-d'Ivoire depuis 1999 et 2011, Madagascar en 2001-2002, Gabon en 2016, RDC en 2011 et 2018, etc.). Les critiques

\footnotetext{
${ }^{1}$ CENTRE CARTER, Obligations et normes électorales : manuel d'évaluation, CENTRE CARTER, Atlanta, 2014, p. 8.

${ }^{2}$ El Hadj MBODJ, «Les garanties et éventuels statuts de l'opposition en Afrique » in mandat, rôles et fonctions des pouvoirs constitués dans le nouveau système politique de la République démocratique du Congo », modules de formation du PNUD, Kinshasa, 2007, p. .40.

${ }^{3}$ Jean du BOIS de GAUDUSSON, «Les élections à l'épreuve de l'Afrique », in Cahiers du Conseil Constitutionnel n 13 (dossier : la sincérité du scrutin) - janvier 2003, p. 1.
} 
des élections africaines se multiplient et, souvent exprimées en termes vifs sinon virulents, instruisent des procès sans appel. N'a-t-on pas pu voir dans les consultations électorales de véritables impostures se réduisant à de « simples formalités administratives dominées par des acteurs politiques se livrant » à un banditisme électoral plutôt qu'à une compétition loyale ${ }^{4}$. $\mathrm{Au}$ côté du développement du processus démocratique en Afrique francophone, il s'est également développé du côté juridique, un droit électoral, qui étale la manière d'exercer le droit de vote, le processus électoral d'un État, son organisation à travers un code électoral, etc. ${ }^{5}$, ce droit électoral, se trouve soutenu par le droit constitutionnel, qui lui, fait référence à l'organisation politique d'un pays, au mode d'acquisition du pouvoir, son exercice et sa fin et, une reconnaissance des droits fondamentaux ${ }^{6}$. Le même droit constitutionnel règlemente surtout l'exercice de la souveraineté nationale et les limites d'un tel exercice où s'émerge la question de la légitimité. Cette dernière se différencie de la légalité qui, néanmoins, doit la créer et la soutenir. Or la légitimité dans nos régimes démocratiques, découle du principe de la représentation : le peuple souverain est représenté par les élus qui exercent ainsi la souveraineté nationale. C'est donc ces deux droits, électoral et constitutionnel, qui donnent compétence au juge constitutionnel de pouvoir connaitre du contentieux électoral, plus spécifiquement le contentieux de l'élection présidentielle et législative nationale. Le droit électoral trouve ses racines dans la constitution, qui garantit les droits civils et politiques ${ }^{7}$. Et encore une fois, plus que cela, la souveraineté nationale et son exercice, autrement dit, l'émergence de l'autorité politique et son encadrement.

Au Congo Kinshasa comme au Gabon, la Cour constitutionnelle connait du contentieux électoral de l'élection présidentielle et législative nationale. De manière plus concrète, la constitution gabonaise, prévoit «que la Cour Constitutionnelle est, entre autres, juge de la régularité des élections. Elle est également l'organe régulateur du fonctionnement des institutions et de l'activité des pouvoirs publics. Aux termes des dispositions des textes suscités la Cour Constitutionnelle ${ }^{8}$ :

- nomme le Président de la CENAP ;

- reçoit le serment des membres du Bureau de la CENAP et de ses démembrements locaux avant leur prise de fonction ;

- connaît du contentieux relatif à l'éligibilité des candidats aux élections ;

\footnotetext{
${ }^{4}$ Jean du BOIS de GAUDUSSON, Op.cit, p.1.

${ }^{5}$ COMMISSION DE VENISE, Le droit électoral, Editions du Conseil de l'Europe, Strasbourg, 2008, p. 5.

6 Jacques Chevallier. «Droit constitutionnel et institutions politiques : les mésaventures d'un couple fusionnel », in $H A L$, Mélanges en l'honneur de Pierre Avril, Montchrestien, p.184.

7 «Introduction au droit électoral », in https://le-politiste.com/introduction-au-droit-electoral/. Consulté le 15/06/2019.

${ }^{8}$ Lire à ce sujet l'article 83 de la Constitution du Gabon de 1991, révisé à ce jour.
} 
- connaît du contentieux relatif aux résultats électoraux ;

- proclame les résultats des différentes consultations électorales ».

En République démocratique du Congo, la Cour constitutionnelle exerce les mêmes compétences en matière électorale. De ce fait, « la Cour constitutionnelle connaît des recours en interprétation de la Constitution sur saisine du Président de la République, du Gouvernement, du Président du Sénat, du Président de l'Assemblée nationale, d'un dixième des membres de chacune des Chambres parlementaires, des Gouverneurs de Province et des Présidents des Assemblées provinciales. Elle juge du contentieux des élections présidentielles et législatives ainsi que du référendum » ${ }^{9}$. L'expérience électorale de ces deux pays de 1'Afrique francophone, démontre que les cours constitutionnelles jouent trois rôles en matière électorale, l'examen du contentieux électoral, la validation des résultats proclamés par la commission électorale nationale indépendante et la proclamation des résultats définitifs des élections présidentielles et législatives. Il est à constater que ces juridictions constitutionnelles sont des actrices importantes de l'alternance démocratique, ayant reçu la mission principale d'entériner le choix du peuple. Toutefois, le bilan ne semble pas être bon, au regard des derniers contentieux électoraux qu'ont connus les cours constitutionnelles du Gabon et de la RD Congo, la tendance est la négation de la souveraineté populaire.

Cette portion de compétence reconnue à la Cour constitutionnelle peut dans certaine circonstance entériner ou se passer du choix du peuple et pour des raisons non élucidées, en proclamant vainqueur, la personne autre que celle qui a été élue par le peuple ${ }^{10}$. Ce qui compliqué davantage encore la tâche, est que, « les arrêts de la Cour constitutionnelle ne sont susceptibles d'aucun recours et sont immédiatement exécutoires. Ils sont obligatoires et s'imposent aux pouvoirs publics, à toutes les autorités administratives et juridictionnelles, civiles et militaires ainsi qu'aux particuliers $»^{11}$.

Ce qui reste une réalité en Afrique francophone, est que « les élections ont été des lieux de contestations entre les acteurs politiques. Ces contestations, conséquences des accusations de fraude et de malversations qui surviennent au lendemain des scrutins, tournent souvent à l'émeute électorale systématique si bien que l'on assiste à des menaces de rupture de la paix

\footnotetext{
${ }^{9}$ Cfr. Art. 161 de la Constitution de la RDC.

${ }^{10}$ Lire à ce sujet les arrêts de la Cour constitutionnelle congolaise intervenus au mois de juin 2019, et qui ont invalidé l'élection de plus de 23 députés de l'opposition (LAMUKA) au profit du camp Kabila (FCC). Le comble est que ces arrêts sont intervenus en violation du délai de la loi électorale qui est de 3 mois après la tenue des élections, ceux-ci, sont intervenus 6 mois après, mais aussi, il y'a une insuffisance presque totale de motivation.

${ }^{11}$ Esprit de l'article 168 de la Constitution congolaise du 18 février 2006, telle que révisée en 2011, in J.O. /RDC, 47 ${ }^{\text {ème }}$ année, $\mathrm{n}^{\circ}$ spécial du 18 février 2006.
} 
sociale étroitement liées aux conditions de légitimité et d'acceptation des résultats des élections et du contentieux électoral $»^{12}$. Ce cycle infernal de contestation électorale, suscite un intérêt de jeter un regard analytique sur les solutions qu'apporte le juge constitutionnel aux questions électorales :

- le juge constitutionnel participe-t-il à une meilleure application du droit électoral dans les États d'Afrique francophone?

- Le contentieux électoral contribue-t-il dans les pays d'étude à la légitimation des élections?

À ces questions, il est judicieux de démontrer, que le juge constitutionnel est le juge électoral des élections présidentielles et législatives nationales, mais l'état actuel de la jurisprudence électorale au Gabon et en RD Congo, reste laconique sur une bonne application du droit électoral (I), en outre, les limites dans l'application du droit électoral abouti dans la plus part de cas à des crises post-électorales (II). En gardant en tête que l'idéal de ce droit électoral et la mission du juge électoral, est de trouver le juste équilibre entre droit et démocratie. Le droit encadre l'expression du peuple pour plusieurs raisons afin de permettre d'abord que conformément à l'idée de la démocratie représentative, la décision de la majorité soit respectée mais aussi pour que les minorités ne soient pas trahies et puissent etre éviter les conflits sociaux qui s'en créeraient. Il sied donc de mentionner que la tâche du juge constitutionnel n'est pas donc simple et demande une critique nuancée.

\section{L'application du droit électoral par le juge constitutionnel au Gabon et en RD Congo}

Il sied de noter qu'en droit public, la compétence est d'attribution. Dans l'architecture judiciaire du Gabon et de la RD Congo, le juge constitutionnel est le juge électoral de l'élection présidentielle et des législatives nationales (A). Toutefois, bien qu'ayant la compétence de ce contentieux électoral, un certain nombre d'éléments limitent l'action du juge constitutionnel (B), ce qui donne lieu à une application mitigée et partisane du droit électoral.

\section{A. La compétence électorale reconnue au juge constitutionnel}

Comme nous l'avons indiqué dans la partie introductive, le juge constitutionnel en RD Congo et au Gabon, est le juge électoral des élections présidentielles et législatives nationales.

\footnotetext{
${ }^{12}$ Jean du BOIS de GAUDUSSON, Op.cit, p. 2.
} 
Dans cette fonction, il juge le contentieux électoral, valide ou invalide les résultats provisoires, et proclame, enfin, le résultat définitif ${ }^{13}$. À la même occasion, il reçoit le serment du gagnant à l'élection présidentielle. Ce qui fait du juge constitutionnel, non seulement un juge du contentieux électoral, mais un véritable acteur important de la continuité de la démocratie et sa pérennisation. De la même manière, l'exercice corrompu de sa compétence, ne peut que ruiner la démocratie. Dans l'exercice de sa compétence, le juge constitutionnel du Gabon et de la RD Congo, connaît certaines limites, qui entachent son action et qui la rend moins performante, par rapport à la volonté réelle exprimée par l'électorat, les exigences de la démocratie et de l'État de droit. Et encore une fois surtout l'équilibre entre ces exigences.

\section{B. Les éléments mettant à mal les actions du juge constitutionnel en matière électoral au Gabon et en RD Congo}

Nous devons admettre avec modestie, qu'un certain nombre d'éléments légaux et illégaux, limitent la valeur de l'action du juge constitutionnel, dans la mise en œuvre du droit électoral. Il s'agit, entre autres, des éléments matériels préalables à la tenue d'une élection (a), le changement du mode de scrutin (b), la fraude électorale (c), la nature même du contentieux électoral (d) et la modalité de désignation des juges constitutionnels (e).

\section{a) Les préalables à la tenue d'une élection (enrôlement des électeurs et mode de scrutin ou son changement)}

Il faut noter que l'élection connaît une préparation forte et minutieuse pour son organisation. Ainsi, il y’a exigence des outils juridiques, car ceux-ci, concourent à la bonne organisation des élections. Il s'agit ici, des lois électorales, qui sont votées au parlement et qui donnent l'essentiel sur la manière dont les élections doivent être organisées dans la globalité. C'est vrai que la cour constitutionnelle a la compétence dans l'examen de la constitutionnalité des lois et, a le pouvoir de déclarer une loi inconstitutionnelle, en raison de tel ou tel grief de non-conformité à la constitution, mais, la réalité dans les pays d'étudiés est autre. En RD Congo, par exemple, la nouvelle loi électorale de 2017, a instauré un recours au seuil légal de représentativité. Il s’agit pour le législateur de 2017 « d'un correctif du système proportionnel des listes, par le regroupement des acteurs et partis politiques en des grandes composantes. Le seuil consiste en un pourcentage de suffrage valablement exprimé déterminé par une norme juridique que chaque

\footnotetext{
${ }^{13}$ Art. 83 de la Constitution du Gabon et 161 de la Constitution de la RD Congo, complétés par les dispositions de lois électorales de deux pays.
} 
liste ou candidat indépendant doit atteindre pour être admis à l'attribution des sièges. Il s'applique au niveau national, provincial, municipal et local, selon qu'il s'agit des élections législatives, provinciales, municipales et locales $»^{14}$.

L'objectif de cette innovation malheureuse a été d'écarter les candidats indépendants et les partis politiques à faible représentation, pour les amener de gré ou de force à intégrer le FCC (Front commun pour le Congo de Kabila), ou de se regrouper dans d'autre plate-forme de l'opposition. Il s'est à git ici, d'une limitation du droit à l'éligibilité des candidats indépendants. Les mêmes causes produisant les mêmes effets, cette loi électorale précitée dispose que «les députés nationaux sont élus au suffrage universel direct, pour un mandat de cinq ans renouvelable, dans les conditions suivantes ${ }^{15}$ :

- le nombre total de suffrages valablement exprimés est déterminé pour l'ensemble du territoire national;

- $\quad$ un seuil de représentativité de $1 \%$ du nombre total de suffrages valablement exprimés est déterminé au niveau national;

- il est attribué d'office un siège au candidat ayant obtenu la moitié de suffrages valablement exprimés dans la circonscription;

- seules les listes des partis et regroupements politiques ou des indépendants ayant atteint ou dépassé ce seuil national de $1 \%$ sont admises à l'attribution des sièges ».

Il est à noter que lors de l'examen de la constitutionnalité de cette loi, la Cour constitutionnelle ne l'a pas frappée d'inconstitutionnelle, pour avoir apporté d'une manière ou d'une autre des limitations à la jouissance des droits politiques, à savoir le droit à l'éligibilité. Cela s'explique par l'absence d'indépendance de ladite cour constitutionnelle vis-à-vis du régime de Kabila. Il s'agissait là, d'une stratégie bien réfléchie, qui a permis au FCC de Kabila de gagner plus de 350 sièges sur 500 sièges à l'Assemblée nationale, en écartant les petits partis politiques et les candidats indépendants et surtout en créant des regroupements politiques prêtant à confusion dans la tête des électeurs, sur leur plate-forme d'appartenance, tels que (AABC, AAA, etc., en RD Congo). Bien sûr que l'on peut trouver des opinions contraires à cette critique, allant dans le sens que la limitation du droit d'éligibilité serait admissible, étant donné que les seuils existent dans d'autres pays, avec comme objectif, d'éviter une polarisation des assemblées élues qui rendraient très difficile la constitution d'une majorité claire même dans le cadre d'une coalition, pourtant nécessaire pour former un gouvernement stable. Cet argument a évidemment son pesant d'or, mais le mobile cherché jadis par le FCC (Frond commun pour le Congo) été

\footnotetext{
${ }^{14}$ Cfr. Exposé des motifs de la loi électorale de 2017 en RD Congo.

15 Art. 118 de la loi électorale de 2017 en RDC.
} 
de se défaire des partis de l'opposition, qui sont en grande partie sans ressource financières suffisantes et, qui ont du mal à avoir une représentativité sur l'ensemble du territoire national, le Congo étant très vaste et enclavé.

Au-delà de la loi électorale qui participe déjà à l'élimination préalable des certains candidats, qui du reste seront absents du contentieux électoral, il y'a aussi, les opérations d'enrôlement des électeurs. L'enrôlement d'électeurs est le prélude de toute élection sérieuse, dans un objectif de constituer la masse électorale, dans ce que l'on appelle fichier électoral. Une fois de plus, le juge constitutionnel ne participe pas à ces opérations, alors que le fichier corrompu pour servir de fraude électorale se construit à ce niveau. La conséquence, est que la cour constitutionnelle, sera amenée à examiner le contentieux électoral, sans avoir la maitrise du fichier électoral, et ne se fiera qu'aux données techniques lui apporté par la CENI, sachant tout naturellement que celle-ci, n'est pas toujours indépendante dans beaucoup d'États d'Afrique francophone, tels que la RDC et le Gabon. La CENI joue dans la plupart de cas le jeu du pouvoir en place et, elle est une institution privilégiée pour la réalisation de la fraude électorale par le parti au pouvoir.

\section{b) Le changement du mode de scrutin}

D. KOKOROKO soutient fort justement que « les lois électorales faussent les résultats du suffrage universel et aboutissent à mettre au pouvoir une majorité parlementaire opposée à la majorité des citoyens. Le moyen le plus utilisé par les pouvoirs politiques est l'adoption de lois constitutionnelles modifiant le mode de scrutin $»^{16}$. En effet, l'adoption des lois constitutionnelles échappe à la compétence du juge constitutionnel africain, sauf au Bénin, au Mali et au Burkina Faso où le juge adopte une interprétation extensive de ses compétences. Les lois constitutionnelles permettent aux chefs d'États en exercice, avec la complicité de leur Parlement, de modifier le mode de scrutin lorsque le contexte politique, économique et social ne leur est pas favorable ${ }^{17}$. Ainsi, en République Démocratique du Congo, pour l'élection du président de la République prévue par la Constitution de 2006, le président Joseph KABILA a remplacé le mode de scrutin à deux tours par un mode de scrutin à un seul tour pour l'élection présidentielle de 2011, ce qui lui a permis d'être élu au premier tour avec 48,9\% des suffrages, devant Etienne Tshisekedi wa Mulumba. Cette modification de la constitution a été obtenue à

\footnotetext{
${ }^{16}$ D. Коковоко, "Les élections disputées : réussites et échec", Pouvoir, Revue d'étude constitutionnelle et politique, $\mathrm{n}^{\circ} 129$, Avril 2009, p. 118.

${ }^{17}$ M. SENE, «Le juge constitutionnel face au défi de la continuité démocratique en Afrique noire francophone », in Presses de l'Université de Toulouse 1 Capitole, 2014, p. 3.
} 
travers une loi constitutionnelle de 2011, par révision de la constitution de 2006, à travers la bénédiction d'un parlement totalement acquis à Kabila ${ }^{18}$.

\section{c) La fraude électorale}

Fabrice Lehoucq définit la fraude électorale comme des « efforts clandestins déployés en violation des règles établies dans le but d'influencer les résultats de scrutins électoraux $\gg{ }^{19}$. Cette fraude relève de la stratégie politique «machiavélique ${ }^{20}$. Il s'agit des actes de truquage dont les auteurs essaient toujours de cacher, nient même en cas de découverte ou de reproche. Ainsi, la clandestinité ou l'intention de cacher et l'illégalité sont deux conditions importantes, pour établir de façon objective le caractère « frauduleux » d'un acte en jeu compétitif comme les élections.

Pour nombreux d'analystes et d'observateurs de la politique africaine, la principale stratégie qu'emploient les partis au pouvoir afin de conserver celui-ci, est la fraude électorale. Ils soutiennent que les partis au pouvoir en Afrique ont développé des techniques de fraude électorale très sophistiquées et subtiles, rendant difficile la détection de certaines pratiques aussi bien par le chercheur que par les observateurs électoraux ${ }^{21}$. Selon une déclaration de Pascal LISSOUBA, l'ancien président congolais, « les régimes africains au pouvoir n'organisent pas les élections pour les perdre $»^{22}$. L'on se pose dès lors la question de savoir si les élections qui se déroulent dans certains pays africains constituent des moyens crédibles et susceptibles de promouvoir l'alternance démocratique et politique.

Daniel Calingaert en illustrant les actes de fraude électorale pense que «le découpage arbitraire de circonscription électorale, réduction ou élargissement illégal ou non consensuel de certaines circonscriptions électorales, la manipulation des listes électorales, le truquage et manipulation des résultat, à travers le bourrage d'urnes, le vote de fantômes, consignes erronées sur l'emplacement des bureaux de vote, la clôture hâtive des opérations de vote avant l'heure de la fin du scrutin, annulation de voix défavorables, rectification des résultats, disparition des urnes défavorables, participent activement à la fraude électorale $»^{23}$. L'ensemble de ces

\footnotetext{
${ }^{18}$ V. NTWALI, Le Rôle de l'opposition politique dans une démocratie représentative : le système multipartiste congolais, Ed. Universitaires Européennes, Berlin, 2015, p. 32.

${ }^{19}$ ISSAKA SOUARE, Les partis politiques de l'opposition en Afrique de l'Ouest et leur quête pour le pouvoir d'Etat : cas du Guinée, Ghana, Benin, Thèse de doctorat en science politique, Université du Québec à Montréal, 2010, p.196.

${ }^{20}$ Fabrice Lehoucq cité par ISSAKA SOUARE, Idem, p.197.

${ }^{21}$ Ninsin, Schedler Calingaert, Case, Beber et Scacoo cités par ISSAKA SOUARE, Op.cit, p. 98.

${ }^{22}$ Pascal LISSOUBA cité par ISSAKA SOUARE, Idem. 197.

${ }^{23}$ Daniel Calingaert cité par ISSAKA SOUARE, Op.cit, p.198.
} 
stratégies a été utilisé aux élections présidentielles et législatives au Gabon en 2016, particulièrement dans la province natale de l'actuel Président de la République du Gabon, Ali Bongo, à savoir la province du Haut-Oogoué, province qui a constitué le bastion du bourrage d'urnes. De la même manière, en RD Congo lors des élections de 2011 dans l'ancienne province du Katanga, ce qui a permis à Kabila de se faire élire massivement dans cette province. En 2018, ces stratégies ont été utilisées, mais les carottes semblaient être cuites pour le régime Kabila, qui n'avait plus de crédibilité aux yeux d'une majorité des Congolais, ce qui a fait que l'opposition gagne les présidentielles et le camp Kabila, les législatives à travers la stratégie de seuil électoral ${ }^{24}$.

Au regard de cette réalité, force est de constater que le juge constitutionnel reste sans outil, face au cheminement procédural et matériel de la fraude électorale, étant donné qu'il n'intervient pas lors des préludes à la tenue des élections. Son pouvoir reste fort au regard de la compétence qui lui est reconnue en matière de contentieux électoral. Malgré cela, il est presque désarmé vis-à-vis de la CENI et des acteurs politiques, surtout ceux du pouvoir sortant, dans la mesure où, dans le règlement du litige, il aura du mal à retracer les éléments ayant constitué le fichier électoral, même dans la logique où, on procéderait à un recomptage des voix, le fichier a considéré ou la masse électorale, sera toujours celle ou celui tenu (e) par la CENI.

\section{d) La nature même du contentieux électoral}

Les principes constitutionnels tels que l'universalité du suffrage, l'égalité des candidats, le droit au vote sont souvent violés sous le regard passif du juge électoral. C'est pourquoi A. FALL soutient qu'il y a « autant d'éléments importants et souvent déterminants quant à l'issue du scrutin, mais qui échappent très souvent au contrôle du juge constitutionnel rarement saisi à leur sujet, alors qu'ailleurs, ils constituent une source importante du contentieux $»^{25}$. Dans la plupart de cas, le juge constitutionnel n'est saisi que du contentieux relatif à la validation ou l'invalidation des résultats, et celui relatif aux candidatures et aux listes de candidatures. Les éléments qui sont aisément et stratégiquement utilisés pour servir à la fraude électorale, ne sont pas dans la pratique en RD Congo et au Gabon, sujets au contentieux électoral, et le juge électoral n'en maitrise pas non seulement le contenu, mais aussi et surtout, les contours. Il s'agit

\footnotetext{
${ }^{24}$ V. NTWALI, Op.cit, p. 26.

${ }^{25}$ A. FALl, "Le processus de démocratisation en Afrique francophone : le juge de l'élection dans l'impasse ?" (Essai de prospective), in Démocratie et élections dans l'espace francophone, Bruxelles, Bruylant, 2010, p. 556.
} 
de l'ensemble des opérations d'enrôlement des électeurs, le découpage des circonscriptions électorales, le fichier électoral, etc.

\section{e) La modalité de désignation des juges constitutionnels}

Mamadou SENE note «qu'une des stratégies utilisées par l'autorité politique pour assurer son succès aux échéances électorales est la maîtrise de la nomination du juge constitutionnel. Ainsi, dans certains États africains, l'autorité politique dispose du monopole du pouvoir de nomination, surtout quand il s'agit de la nomination d'un président d'une Cour ou d'un Conseil constitutionnel, les cas sénégalais et ivoiriens l'attestent $»^{26}$. Ce monopole de la nomination permet aux pouvoirs politiques de faire du juge constitutionnel une marionnette qui entérine toutes les manœuvres frauduleuses. Il engendre un discrédit du juge constitutionnel et il constitue une entrave à son action ${ }^{27}$. De ce fait, il est rare, en Afrique francophone, de voir une cour constitutionnelle, qui invalide l'élection d'un candidat au pouvoir, la validation pure et simple est toujours la tendance. Du côté anglophone de l'Afrique, rappelons qu'en 2017, une sorte d'éveil du juge constitutionnel du Kenya s'était affirmé, étant donné que la cour constitutionnelle kenyane avait rejeté le résultat provisoire de la CENI, ayant placé Uhuru Kenyatta en tête devant Railla Odinga, en appelant au recomptage des voix. Mais curieusement, c'est le même Uhuru Kenyatta qui a été proclamé définitivement comme gagnant, ce qui a donné lieu à une grave crise post-électorale.

\section{L'interprétation laconique du droit électoral par le juge constitutionnel au Gabon et en RD Congo : gage des crises post-électorales}

L'examen du point I de cette étude, permet de constater que le juge constitution dans la pratique au Gabon et en RD Congo, exerce une compétence limitée. Sur l'ensemble du contentieux du processus électoral, celui-ci ne parvient pas à faire une bonne application du droit électoral, étant de manière pratique en situation de manque des preuves, ce qui l'expose habituellement à la corruption. Son action dans les pays d'étude précités, semble se limitée aux

\footnotetext{
${ }^{26}$ M. SENE, Op. cit., p. 5.

${ }^{27}$ Au Sénégal, tous les membres du Conseil constitutionnel sont nommés par le président de la République ; au Burkina Faso et au Gabon, le pouvoir de nomination est partagé entre les autorités politiques, notamment le président de la République, le président de l'Assemblée Nationale, le président du Sénat. Au Burkina Faso, aux membres nommés s'ajoutent les anciens présidents de la République. En République démocratique du Congo, le président de la République nomme seul 3 juges, le parlement 3 et 3 autres sont nommés par le Conseil supérieur de la magistrature. Cela fait que déjà le président a la majorité au parlement et les 3 juges qui y sont nommés, lui sont aussi favorables, ce qui fait automatiquement qu'il a déjà 6 juges sur 9 qui lui sont favorables. L'on doit noter que même ceux qui sont désignés par le Conseil supérieur de la magistrature, n'échappent pas à cette règle, étant entendu bien évidemment que les magistrats du Conseil supérieur de la magistrature sont nommés par ordonnance présidentielle. On assiste donc à des cours constitutionnelles inféodées au pouvoir.
} 
contentieux nés des opérations de vote le jour du scrutin et les résultats affichés par la CENI, sachant que la CENI, est, dans la plupart de cas, corrompue

Le développement de ce deuxième point repose sur le contentieux électoral de 2016 au Gabon, et celui de 2011 et 2018 en RD Congo. Ainsi, la démarche consiste à jeter, en premier, un regard sur les tendances jurisprudentielles du juge électoral au Gabon et en RD Congo (A), ce qui prouvera l'application limitée du droit électoral, avant d'examiner les conséquences de ces tendances jurisprudentielle sur la confiance des acteurs et la stabilité socio-politique des deux pays d'étude. Enfin, nous verrons s'il y'a une réponse constitutionnelle à l'application lacunaire du droit électoral, réservée au peuple pour sauver sa volonté exprimée lors d'un vote (B).

\section{A. Tendances jurisprudentielles du juge électoral au Gabon et en RD Congo}

Comme indiqué dans la partie introductive, notre analyse ne concerne que le contentieux des élections présidentielles et législatives nationales dans les deux pays d'étude.

\section{Le juge électoral gabonais face à la requête de Jean Ping du 8 septembre 2016}

Le développement de ce point nécessite une étude de cas, ce qui fait qu'il évoque en premier le contenu de la requête, la position de la Cour et enfin, une observation.

\section{a) Contenu de la requête de Jean Ping}

Il est judicieux de rappeler que la saisine de la Cour par Jean Ping, ne semble pas avoir été faite dans la joie, elle est intervenue après beaucoup d'hésitations liées à une certaine manque de confiance du requérant vis-à-vis de la Cour constitutionnelle. Il a « attendu le dernier moment mais il n'a pas reculé. L'opposant à Ali Bongo a déposé, jeudi 8 septembre 2016, un recours devant la Cour constitutionnelle du Gabon pour contester les résultats de l'élection présidentielle du 27 août 2016, remportée par le président sortant Ali Bongo Ondimba. Sa principale demande consistait au recomptage des voix dans la province du Haut-Oogoué, bureau de vote par bureau de vote, à travers la confrontation des procès-verbaux détenus par la 
Cenap (Commission électorale nationale) et toutes les parties $»^{28}$. À rappeler que cette province est le fief familial d'Ali Bongo, dans laquelle il a obtenu 95\% des voix avec 99,99\% de taux de participation, chiffres qui sont difficilement réalisables dans une démocratie multipartiste.

\section{b) Position de la Cour}

La décision n50/CC du 23 septembre 2016 peut être résumée en ces termes :

«Article premier : les requêtes présentées par messieurs Gérard ELLA MINTHOGHE et Jean PING sont recevables en la forme.

Article 2 : Lesdites requêtes sont rejetées quant au fond.

Article 3 : La demande reconventionnelle formulée par monsieur Ali BONGO ONDIMBA est recevable.

Article 4 : Les résultats de 21 bureaux de vote du 2ème arrondissement de la commune de Libreville, province de l'Estuaire, sont annulés.

Article 5 : En dépit de cette annulation, Monsieur Ali BONGO ONDIMBA conservation toujours une avance sur les autres candidats.

Article 6 : La présente décision sera notifiée aux parties, au Premier Ministre, au président du Sénat, au président de l'Assemblée Nationale et publiée au Journal officiel de la République gabonaise ou dans un journal d'annonces légales

Est proclamé élu :

Ali Bongo Ondimbal72 990 voix, 50,66\% $»^{29}$.

\section{c) Observations :}

La décision précitée de la Cour soulève, à notre avis, deux observations capitales pour matérialiser l'application mécanique du droit électoral par le juge constitutionnel gabonais : $\mathbf{1}^{\text {érè }}$ observation : nous fondant sur les arguments de parties, Ping, Bongo et la position de la Cour, nous constatons rapidement que pour l'annulation des résultats de 21 bureaux du deuxième arrondissement de Libreville, qui du reste étaient favorables au requérant premier Jean Ping, la Cour s'est plutôt fondée sur les procès-verbaux prétendument raturés par Ping et présentés à l'occasion du procès par Ali Bongo. Ce qui est aberrant, la Cour n'a pas sollicité les procès-verbaux de la CENAP, qui elle, devait dire la vérité à la Cour en lui présentant l'ensemble des procès-verbaux à la forme originale et signés par tous les témoins et

\footnotetext{
28 « Jean Ping saisit la Cour constitutionnelle pour contester les résultats de la présidentielle », disponible sur https://www.france24.com/fr/20160908-gabon-mediation-union-africaine-reportee-sine-die-nouvel-ordre-ping-bongo. Consulté le 17/06/2019.

${ }^{29}$ Décision n50/CC du 23 septembre 2016 rendue par la Cour constitutionnelle du Gabon dans la requête en reformation des résultats de l'élection présidentielle introduite par Jean Ping.
} 
observateurs, pouvant, à notre avis, éclairer la mémoire de la Cour. Ce qui consolide encore une fois de plus la démarche limitée de la Cour, est que si les fameux procès-verbaux raturés, présentés par Ali Bongo, avaient été les vrais, la CENAP n'en aurait pas tenu compte dans les procès-verbaux de proclamation des résultats provisoires. Or, en aucun moment la CENAP n'a fait part de ces fameux procès-verbaux raturés et surchargés ${ }^{30}$.

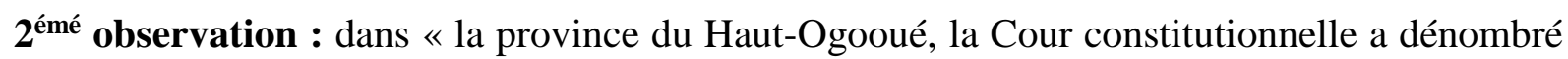
et proclamé 71642 inscrits, 70660 votants, 314 bulletins blancs ou nuls, 70346 suffrages exprimés et $98,62 \%$ de taux de participation ${ }^{31}$. Pour les avocats de Ping, « ces résultats ont à l'évidence été calculés sur la base de fausses pièces ». Sinon comment comprendre que selon les procès-verbaux authentiques en possession de Jean Ping, la commune de Moanda à elle seule totalise 454 bulletins blancs ou nuls et si l'on ajoute celle de Mounana où on en compte 147, cela fait déjà 601 bulletins blancs ou nuls ; alors que la Cour constitutionnelle n'en dénombre que 314 pour toute la province du Haut-Ogooué $»^{32}$. Nous constatons que la Cour a procédé au recomptage des voix bureau de vote par bureau de vote, en tenant seulement compte des procès-verbaux de la CENAP, alors que celle-ci, n'a pas joué un jeu d'impartialité et de neutralité. Il y'a eu donc absence d'une confrontation des procès-verbaux détenus par les deux parties et ceux de la CENAP, dans la démarche de la Cour. Cela justifie la différence entre les chiffres trouvés par la Cour, par rapport aux résultats contenus dans les procès-verbaux détenus par les parties. En définitive, le recours de Jean Ping n'a pas bouleversé la tendance par rapport à l'ordre d'arriver des candidats mais, par la démarche peut orthodoxe de la Cour, ledit recours a permis l'annulation des procès-verbaux favorables à Ping, ce qui a diminué son pourcentage à 47,24\% et l'augmentation du pourcentage d'Ali Bongo de 49\% à 50,6\%.

En toute impartialité et dans un sens d'objectivité, la Cour aurait dû examiné « le dossier lui soumis par la CENAP qui comprenait outre les procès-verbaux des bureaux de vote, ceux des commissions électorales départementales, communales, provinciales, diplomatiques et consulaires ainsi que le procès-verbal de centralisation établi par la CENAP, en les confrontant, à ceux détenus par les parties $»^{33}$. Une telle démarche aller un tant soit peu, donner du crédit à

\footnotetext{
${ }^{30} \mathrm{Cfr}$. Considérant 51 de la Décision n50/CC du 23 septembre 2016 rendue par la Cour constitutionnelle du Gabon dans requête en reformation des résultats de l'élection présidentielle introduite par Jean Ping.

${ }^{31}$ Ibid.

${ }^{32}$ Lire à ce sujet la requête de Jean Ping en révision de la Décision n50/CC du 23 septembre 2016 rendue par la Cour constitutionnelle du Gabon dans requête en reformation des résultats de l'élection présidentielle introduite par Jean Ping.

${ }^{33}$ Requête en révision de Jean Ping, Op. cit., p. 2.
} 
la décision de la Cour, mais hélas, les intérêts semblent avoir été autres, qu'une application orthodoxe du droit électoral gabonais. Le juge électoral congolais, a-t-il fait le contraire ?

\section{Le juge électoral congolais face au contentieux de 2011 et 2018}

Pour illustrer l'approche du juge constitutionnel congolais au contentieux électoral de l'élection présidentielle et de législative nationale, nous allons faire recours à deux arrêts. Il s'agit de l'arrêt sous R.E 007 du 22 décembre 2011 en contestation des résultats provisoires proclamés par la CENI pour les élections présidentielles et législatives du 28 novembre 2011 et l'arrêt RC 001/PR rendu le 19 janvier 2019 sur la requête en contestation des résultats de l'élection présidentielle et législative du 30 décembre 2018 de l'opposant Martin Fayulu. Ces deux arrêts démontrent également l'application laconique, mécanique et partisane du droit électoral par le juge constitutionnel congolais.

a) Contenu de la requête de l'Union pour la nation congolaise (UNC) de Vital Kamerhe

Par sa requête datée du 12 décembre 2011, introduite devant la Cour suprême de justice faisant office de la Cour constitutionnelle, l'Union pour la nation congolaise de Vital Kamerhe, avait saisi ladite Cour en contestation des résultats de l'élection présidentielle du 28 novembre 2011. À l'appui de sa requête, la requérante soutenait « que le processus électoral qui a conduit à la proclamation des résultats provisoires contestés était émaillé de plusieurs irrégularités, ayant influé de manière déterminante sur ceux-ci $»^{34}$.

Elle prétendait ainsi «d'une part que la loi électorale a été violée à savoir l'article 8 par la CENI, à travers les actes suivants : non publication des listes des électeurs provinces par province avec leurs identités complètes 30 jours avant la date du scrutin, la CENI l'ayant fait seulement deux jours avant la date du scrutin, ce qui a participé au faible taux de participation, les bulletins de vote ne prévoyant pas de place pour la signature de plus de 5 témoins dans le bureau de vote, le non-accompagnement du transfert des procès-verbaux des centres de vote au bureau de compilation par les témoins de l'UNC, à travers les manœuvres de la CENI, l'interdiction d'accès au centre national de traitement des résultats pour les témoins UNC, d'autre part, que les résultats proclamés par la CENI manquaient de sincérité et méritaient annulation en raison de la circulation entre les mains de caciques du MP des bulletins de vote déjà cochés en faveur de Kabila, le cas de Néron Mbungu : des bulletins de vote insuffisants par rapport au nombre d'électeurs enregistrés dans les provinces acquises à l'opposition,

\footnotetext{
${ }^{34}$ CSJ, Arrêt R.E 007 du 28 décembre 2011.
} 
l'exemple du Sud-Kivu et le Nord-Kivu : résultats provisoires de la CENI non conformes aux résultats publiés après le dépouillement dans les bureaux de vote $»^{35}$. En lisant la requête de l'UNC, l'on constate qu'elle avait fait un effort considérable dans l'énumération de l'ensemble d'actes de violation de la loi électorale et la fraude électorale au profit du candidat Kabila. Toutefois, dans son arrêt la Cour est totalement passé à côté.

\section{b) La position de la Cour}

La Cour dans sa motivation, soutient que « ne doivent être retenues que les irrégularités susceptibles de fausser les résultats de l'élection, eu égard notamment à l'écart des voix entre les candidats. Il est dès lors évident, soutient la Cour, que la simple violation de la loi n'entraine pas nécessairement l'annulation de l'élection, le juge pouvant confirmer celle-ci, s'il estime non seulement que les faits allégués ne sont pas de nature à modifier le résultat, en dépit d'une irrégularité constatée dans le déroulement de la campagne électorale ou des opérations électorales $»^{36}$. En outre poursuit la même Cour dans sa motivation, «les moyens de preuve qu'elle prend en compte dans l'appréciation de la régularité du scrutin sont principalement le procès-verbal de dépouillement, les observations des membres du bureau de vote ou des témoins des candidats, le constat des irrégularités qu'elle aurait relevées par elle-même, ainsi que les réclamations des électeurs annexées aux procès-verbaux $»^{37}$.

Relativement à la violation de la loi électorale, la Cour argue que « le requérant n'apporte pas la preuve démontrant à qui ladite violation aurait profité, de même, la requérante ne démontre pas en quoi ladite violation à travers une preuve, aurait influé sur le taux de participation. Elle poursuit en indiquant que quand bien même la violation aurait été rapportée, il est clair que les violations susvisées de la loi électorale, n'auraient eu aucune incidence sur les résultats du scrutin eu égard à l'écart des voix entre les candidats ». Curieusement sur le $100 \%$ des suffrages exprimés dans la circonscription électorale de Malemba-Nkulu et dont Kabila a renflé en totalité, la Cour a relevé qu'il n'est pas nullement interdit à un candidat à une élection de remporter l'ensemble des voix des électeurs, dans le respect des lois et procédures en la matière ». En plus, concernant les résultats revendiqués par la requérante dans le NordKivu, le Sud-Kivu et l'Ituri, provinces totalement acquises à l'opposition, la Cour a souligné

\footnotetext{
${ }^{35}$ Lire à ce sujet requête de l'UNC en contestation des résultats provisoires de la CENI à l'élection présidentielle du 28 novembre 2011.

${ }^{36}$ Cfr. CJS, Arrêt R.C.E. PR.009 du 27 novembre 2006 et l'arrêt R.E. 007.

${ }^{37}$ Ibid.
} 
« qu'ils ne sont confirmés par aucun élément de preuve, les vérifications opérées confirmant plutôt des chiffres publiés par la CENI ». En définitive, la Cour a jugé le recours de l'UNC non fondé et par conséquent, elle a proclamé Kabila Joseph gagnant du présidentielle de 2011.

c) Observations

Deux observations sautent également aux yeux par rapport à cette interprétation lacunaire de la Cour :

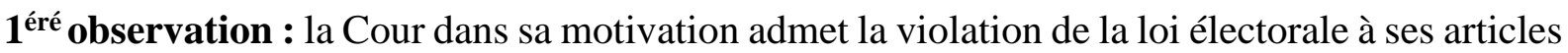
8, 56, 48 mais refuse d'appliquer les sanctions, qui devaient impérativement aboutir à l'annulation des résultats proclamés par la CENI. Elle adopte, par contre, une démarche partisane en soutenant abusivement «que même si la preuve a été rapportée, les violations de la loi électorale n'auraient eu aucune incidence sur le résultat du scrutin, eu égard à l'écart des voix entre les candidats ». La Cour semble soutenir ici l'écart entre candidats, comme moyen pouvant couvrir les violations de la loi électorale, oubliant que ces violations de la loi, ont été à l'occasion de la construction frauduleuse des résultats évoqués. Ce qui est encore grave, ladite Cour souligne encore que «les faits allégués ne sont pas de nature à modifier le résultat, en dépit d'une irrégularité constatée dans le déroulement de la campagne ou des opérations électorales ». Ici la Cour admet également l'existence des irrégularités, mais on ne sait pas par quelle magie, elle affirme que ces irrégularités ne sont pas de nature à modifier l'ordre d'arriver des candidats. Il s'agit ici, d'une contradiction totale, dénotant une interprétation laconique du droit électoral par le juge électoral congolais.

$2^{\text {émé }}$ observation : Alors qu'elle rappelle «qu'en matière de contentieux des résultats électoraux, le juge électoral vérifier l'authenticité et la sincérité du scrutin, en cherchant les incidences des irrégularités constatées sur les résultats ${ }^{38}$, elle s'est tout de même, limité à fonder son argument sur les seuls procès-verbaux de la CENI, sur la base desquels, les résultats contestés ont été fabriqués, en mettant de côté les preuves de la requérante. Elle aurait dans une démarche objective, faire une confrontation des procès-verbaux ou du moins, procéder au recomptage des voix. À l'opposé, elle a préconisé aller sur la défensive, en soutenant «qu'il n'est pas interdit à un candidat de remporter l'ensemble des voix des électeurs soit $100 \%$ du suffrages exprimés ${ }^{39}$, sans se poser la question de savoir s'il n'y a pas eu d'abstention, des bulletins nuls, ou des voix favorables à l'opposition, dans la mesure où, il n'est pas facile de réaliser un score de 100\% dans une démocratie. Qu'en est-il de l'arrêt Fayulu devant la même Cour constitutionnelle?

\footnotetext{
${ }^{38}$ Arrêt R.C.E. PR.009 du 27 novembre 2006 et l'arrêt R.E. 007, Cour suprême de Justice.

${ }^{39}$ Ibid. .
} 


\section{Contenu de la requête de Fayulu (Dynamique de l'opposition) candidat de Lamuka}

L’opposant Martin Fayulu a déposé son recours devant la Cour constitutionnelle contre les résultats proclamés par la CENI à l'élection présidentielle et législative du 30 décembre 2018, qui ont placé Felix Tshisekedi du Cap pour le changement $(\mathrm{CACH})$ en tête avec $38 \%$, suivie du requérant Martin Fayulu de la plate-forme Lamuka avec 34\% et avec 24\% pour le dauphin de Kabila, Emmanuel Ramazani Shadary ${ }^{40}$. Ledit recours a été enregistré sous RCE 01/PR, et visé l'annulation des résultats proclamant Felix Tshisekedi président de la RD Congo, le requérant arguant qu'il fallait procéder au recomptage des voix, étant donné que les résultats contestés de la CENI, n'étaient pas conformes à la vérité des urnes, et étaient totalement contraires au résultat recueilli par la mission d'observation de la CENCO qui mettait en tête Fayulu avec plus de $60 \%$ de voix.

\section{a) Position de la Cour}

Par son arrêt sous RC 001/PR du 19 janvier 2019, la Cour constitutionnelle a rejeté la requête de Martin Fayulu, la déclarant non-fondée ${ }^{41}$. La Cour s'est fondée sur les procèsverbaux déposés par la CENI, soutenant que le requérant n'avait pas apporté des preuves soutenant ses allégations lui plaçant en tête de la présidentielle du 30 décembre 2018, avec plus de $61 \%$ de voix.

\section{b) Observations}

Le contour de cette affaire, soulève deux observations.

$\mathbf{1}^{\text {éré }}$ observation : au niveau de la Cour et les arguments de sa motivation, il sied de noter que celle-ci, n'a fondé son arrêt que sur les fichiers de centralisation des résultats de la CENI, qui sont électroniques et que la loi électorale ne prévoit pas, et les procès-verbaux de la CENI. À noter que la Cour n'a pas procédé au recomptage des voix, tel que solliciter par la requérante de la Dynamique de l'opposition. Ce qui a fait que sans aller plus loin, la Cour n'a fait que confirmer les résultats provisoires proclamés par la CENI, alors que ces élections connaissaient des nombreuses contestations de la part des acteurs et des observateurs ayant participé à ces élections.

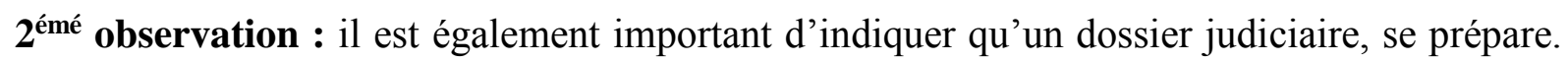
Au regard du déroulement de l'affaire sous RCE 01/PR, il est vrai que l'opposant Martin Fayulu

\footnotetext{
40 «Contentieux électoral en RDC : la Cour constitutionnelle examine la requête de Fayulu », disponible sur https://fr.africanews.com/2019/01/14/contentieux-electoral-en-rdc-la-cour-constitutionnelle-examine-la-requete-de//. Consulté le 20/06/2019.

${ }^{41} \mathrm{CC}, \mathrm{RC} 001 / \mathrm{PR}$ du 19 janvier 2019.
} 
n'a pas bien préparé son dossier de recours, surtout en ce qui concerne les preuves. Il sied de rappeler qu'en droit l'allégation d'un fait, nécessité nécessairement une preuve à ce sujet et dans le cadre de cette affaire, le requérant Fayulu de la Dynamique de l'opposition, allégué avoir gagné les élections à plus de 60\%, mais devant la Cour constitutionnelle, ce dernier n'est pas parvenu à prouver ses affirmations, étant donné qu'il n'avait pas des preuves, dans le cas d'espèce, les procès-verbaux de bureaux de vote. Cela s'explique par le fait que la plate-forme Lamuka, qui portait Fayulu à la présidentielle de 2018 en RD Congo, n'avait pas affecté des témoins dans tous les bureaux de vote au niveau de l'ensemble du territoire national. Les allégations du requérant, sont restées des affirmations gratuites sans aucune preuve matérielle, se fondant uniquement sur le rapport d'observation de la $\mathrm{CENCO}^{42}$, alors que celle-ci, avec le statut d'observateur des élections, n'a pas de compétence légale, en RD Congo, de détenir les procès-verbaux des bureaux de vote.

Toutefois, il sied de noter que le juge constitutionnel étant le juge du contrôle de la régularité et de la sincérité de l'élection présidentielle et législative nationale, aurait dû, procéder au recomptage des voix, cela donnerait encore une fois de plus une crédibilité aux élections du 30 décembre 2018 et, une sorte de légitimité pour le gagnant. Sa position dans cette affaire, n'a fait qu'alimenter une crise post-électorale.

\section{B. Le mécanisme constitutionnel du rétablissement de la vérité des urnes}

L'examen de ces trois recours en contestation des résultats des élections présidentielles et législatives au Gabon en 2016 et en RDC en 2011 et 2018, permet de constater que dans l'application du droit électoral, les juges électoraux dans ces deux pays étudiés, sont simplement à la commande du pouvoir, ce qui fait qu'ils n'appliquent pas correctement le droit électoral, étant totalement inféodés à l'exécutif sortant. Ces juges bénéficient de la valeur juridique et la force obligatoire immédiate que la constitution accorde aux arrêts de la Cour constitutionnelle ${ }^{43}$.

\footnotetext{
${ }^{42}$ Selon le rapport de la CENCO (Commission Episcopale nationale du Congo) « A chaque fois, c'est le même candidat qui arrive en tête : Martin Fayulu loin devant sur chacun des comptages, avec 62\% des voix sur l'échantillon le plus large, puis Félix Tshisekedi deuxième et Emanuel Ramazani Shadary troisième avec, sur ce même échantillon, respectivement 16,88\% et $16,93 \%$ des voix. Un écart de voix très important sépare donc le premier du deuxième, trop important pour être rattrapable même en incluant les près de $29 \%$ de votants restants dont les voix n'ont pas été décomptées par la CENCO ». Ces chiffres sont basés sur un échantillon de vérification des résultats sur 71,53\% des votes, et donc, la CENCO n'a pas eu connaissance de résultat de $29 \%$ de vote. La grande difficulté est que légalement la CENCO ne pouvait pas avoir en possession des procèsverbaux de vote de chaque bureau, étant un simple observateur. Elle a, toutefois, recueilli ses informations, à travers des Smartes phones reçus de l'UE, pour aller plus loin, visiter http://www.rfi.fr/afrique/20190118-elections-rdc-rapport-detaille-cencoresultats-ceni. Consulté le 21/06/2019.

${ }^{43}$ A ce sujet, l'article 168 de la Constitution de la RDC dispose que « Les arrêts de la Cour constitutionnelle ne sont susceptibles d'aucun recours et sont immédiatement exécutoires. Ils sont obligatoires et s'imposent aux pouvoirs publics, à toutes les
} 
Rappelons que dans les deux pays étudiés, les arrêts de la cour constitutionnelle ne font l'objet d'aucun recours, s'exécutent immédiatement et s'imposent à tous. Toutefois, il est important de relever, que la Constitution, qui prévoit la Cour constitutionnelle et ses compétences en matière électorale tout comme dans d'autres matières, contiendrait, en plus, un certain nombre de mécanisme capable de rétablir la vérité des urnes, face à une Cour constitutionnelle totalement inféodée. Il s'agit de la voie révolutionnaire et de la voie institutionnelle.

\section{Le rétablissement de la vérité des urnes par la voie révolutionnaire}

Devant un push électoral, entériné par les juges de la Cour constitutionnelle, la constitution congolaise, par exemple, a prévu la voie révolutionnaire comme remède. Ainsi, son article 64 consacre un «droit à la révolution ou à la rébellion ${ }^{44}$ contre toute forme de dictature. Selon André Mbata, cet article « se serait inspiré de la Conférence nationale souveraine et la Constitution du Bénin. Il est l'un de plus importants articles de la Constitution congolaise $»^{45}$. Pour lui, « contrairement à l'interprétation étriquée qui en est généralement donnée, cet article est de nature à s'appliquer au Président de la République, au gouvernement (national, provincial ou local), au parlement (national ou provincial), et à tout service public qui exerceraient le pouvoir en violation des dispositions de la Constitution $»^{46}$. L'on doit donc savoir que la Constitution n'a pas octroyé un «pouvoir absolu aux juges » même si les arrêts de la Cour ne sont susceptibles d'aucun recours et sont immédiatement exécutoires ${ }^{47}$. La Constitution a établi un gouvernement du peuple, qui seul est souverain et exerce cette souveraineté par voie des élections libres, régulières et transparentes ou par voie de référendum, il serait donc inacceptable, qu'une Cour constitutionnelle, s'inscrive à contre-courant du choix électoral exercé par le peuple.

De ce fait, à la recherche de la vérité des urnes expression de sa volonté électorale, «le peuple pourrait aussi lui brandir l'article 64 si ses membres exerçaient leur pouvoir en

\footnotetext{
autorités administratives et juridictionnelles, civiles et militaires ainsi qu'aux particuliers ", il s'agit d'une même formulation avec l'article 92 de la Constitution du Gabon qui prévoit également que «Les décisions de la Cour constitutionnelle ne sont susceptibles d'aucun recours. Elles s'imposent aux pouvoirs publics, à toutes les autorités administratives et juridictionnelles et à toutes les personnes physiques et morales ». Cette valeur juridique accordée par la constitution aux arrêts de la Cour constitutionnelle, devrait appeler les juges constitutionnels et électoraux à plus de responsabilité, en faisant une application orthodoxe de la Constitution et de la loi électorale.

${ }^{44}$ Art. 64 al. 1 de la Constitution de la RD Congo du 18 février 2006.

45 A. MBATA, «Arrêt de la Cour constitutionnelle : nomination des commissaires spéciaux, respect de la Constitution, l'élection présidentielle, de la Cour constitutionnelle et de l'article 64 », in Social Science Research Council, New York, 28 avril 2016, pp. 2-6.

${ }^{46} \mathrm{Ibid}$.

${ }^{47}$ Esprit de l'art. 5 de la constitution congolaise du 18 février 2006, telle que révisée en 2011.in J.O/RDC, $47^{\text {ème }}$ année, $n^{\circ}$ spécial du 18 février 2006.
} 
violation de la Constitution en interprétant de manière tendancieuse les dispositions de la constitution et en appliquant de manière partisane le droit électoral, pour changer la vérité des urnes exprimée par le peuple. À notre avis, un tel arrêt serait un "coup d'État contre la Constitution et la démocratie » qui serait l'œuvre de la Cour et qui appellerait la même réponse que le peuple réserverait à tout coup d'État ${ }^{48}$. Le peuple a aussi le devoir de s'opposer à une Cour constitutionnelle qui se réduirait à un groupe d'individus ou de putschistes contre la Constitution et la vérité des urnes exprimée par le peuple. Pour A. Mbata, « ce serait le cas, par exemple, dans nos pays où toutes les folies sont possibles au nom du pouvoir et de l'argent, si la Cour constitutionnelle décidait de transformer les hommes en femmes ou le contraire en se fondant sur le fait que ses arrêts sont sans recours ! Personne n'obéirait à un tel arrêt ${ }^{49}$.

Il convient de signaler, comme le mentionne bien le Professeur André Mbata que «par rapport à cette situation que l'histoire récente de la RDC est jalonnée des crises politiques nées des difficultés d'interprétation des textes juridiques ${ }^{50}$. Certains de juristes congolais même ceux appartenant à des institutions publiques telles que les universités, la Cour constitutionnelle, la Cour Suprême de Justice et le Parquet général de la République font, de temps en temps, preuve des insuffisances quant à l'interprétation de la Constitution. De plus, les difficultés d'interprétation accroissent lorsque la disposition en question protège un intérêt politique donné ou elle le menace $»^{51}$. Tel est le cas du droit électoral, qui une fois violé, sans application

\footnotetext{
${ }^{48}$ A. MBATA, Op. cit., pp. 2-6.

${ }^{49}$ Ibid.
}

${ }^{50}$ A. MBATA note qu'en 1960, le Chef de l'Etat Joseph KasaVubu révoqua le Premier ministre Patrice Emery Lumumba sur fond d'une interprétation erronée de l'article 22 de la Loi fondamentale. Trente-cinq ans après, la famille politique du Président Mobutu et l'Union sacrée de l'opposition s'opposaient quant à la compréhension de l'article 78 de l'ACT du 9 avril 1994. La Constitution de transition du 05 avril 2003 a engendré des controverses autour de deux articles. Il s'agit de l'article 76 relatif à la nomination des hauts fonctionnaires de l'État, des gouverneurs et vice-gouverneurs de province, des gouverneurs de la BCC, des ambassadeurs, des magistrats et des mandataires publics. L'article 196 concernait le délai de la transition. Cette disposition stipulait que «La durée de la transition est de vingt-quatre mois. Elle court à compter de la formation du Gouvernement de transition et prend fin avec l'investiture du Président de la République élu à l'issue des élections marquant la fin de la période transitoire en République Démocratique du Congo. Toutefois, en raison de problèmes spécifiquement liés à l'organisation des élections, la transition peut être prolongée pour une durée de six mois renouvelable une seule fois, si les circonstances l'exigent, sur proposition de la Commission électorale indépendante et par une décision conjointe et dûment motivée de l'Assemblée nationale et du Sénat ». Les controverses nées à l'occasion de l'interprétation de cette disposition sont résumées par Minani Buhuzo R, «République Démocratique du Congo : La démocratie à l'épreuve de la géopolitique et la bonne gouvernance », Congo-Afrique, $\mathrm{n}^{\circ} 423$, mars 2008, pp. 220-221. Il notait en ce sens que « Les querelles dues à l'insatisfaction des uns et des autres dans la gymnastique compliquée du 'partage équitable et équilibré des responsabilités', relégué le chronogramme des élections au second plan. C'est cet état de choses qui a fait que le calendrier de la transition soit lui-même élastique. Deux ans n'ont pas suffi à faire aboutir la période de transition. Cette question du délai constitutionnel de la fin de la transition a d'ailleurs alimenté le débat politique et menacé la paix sociale. Les acteurs politiques ont souvent adopté un double langage. Tout en prétendant vouloir des élections dans l'immédiat, ils essayaient, à chacune de ses phases, d'en prolonger le délai, de réorienter le processus, de le requalifier ou tout simplement, de le saboter ».

${ }^{51}$ Cfr Arrêt (R.A. 320) du 12 août 1996 dans l'affaire USOR. Dans le meme sens, Mbata Betukumesu Mangu A., The Road to Constitutionnalism and Democracy in Post-colonial Africa.The Case of Democratic Republic of Congo, Doctoral Thesis, 
correcte par la Cour constitutionnelle (ici juge électoral), ne porterait directement atteinte aux intérêts du régime sortant, celui-ci, usant, dans la plupart de cas, de la fraude électorale.

Relativement au Gabon, ce mécanisme de révolution est prévu à l'article 1 point 21 de la constitution gabonaise, qui prévoit que «chaque citoyen a le devoir de défendre la patrie et l'obligation de protéger et de respecter la Constitution, les lois et les règlements de la République ${ }^{52}$. Sur base de cet article 1 point 21 de la Constitution gabonaise, à l'instar de l'article 64 de la constitution congolaise, le peuple à travers une révolution peut aboutir au rétablissement de la vérité contre un push électoral entériné par le juge électoral. L'exercice d'une révolte par le peuple, peut pousser le juge électoral à une rectification d'erreur matérielle ou d'interprétation.

\section{La rectification d'erreur matérielle (RDC) et procédure en révision (Gabon)}

Étant une œuvre humaine, la justice électorale est confrontée à l'exemple de la justice pénale, civile, etc., à des erreurs matérielles ou d'interprétation, ce qui fait que l'arsenal juridique de la RD Congo et celui du Gabon, a prévu une possibilité pour le juge électoral, de revenir sur son œuvre et de pouvoir corriger les erreurs matérielles ou liées à une interprétation erronée. De ce fait, l'article 93 al. 4 de la loi portant organisation et fonctionnement de la Cour constitutionnelle en RDC, dispose que «les arrêts de la Cour ne sont susceptibles d'aucun recours, sauf interprétation ou rectification d'erreurs matérielles $»^{53}$. Au Gabon, c'est par contre une procédure en révision qui est prévue à ce sujet. Ainsi, l'article 87 de la loi organique sur la Cour constitutionnelle dispose que la révision n'est ouverte que dans les cas suivants : « s'il y a eu fraude de l'une des parties de nature à avoir déterminé la conviction de la Cour ; s'il y'a eu faux témoignage reconnu par une décision de justice, si la décision considérée a été rendue sur des pièces fausses, si, depuis la décision, il a été recouvré des pièces décisives détenues par l'adversaire. Le recours est exercé dans le délai de quinze jours à compter de la notification de la décision attaquée ${ }^{54}$. Toutefois, il est important de mentionner que l'exercice de cette procédure en révision, dans le cadre du droit Gabonais, n'est pas suspensif de l'exécution de l'arrêt attaqué. Voilà donc deux moyens que la victime du push électoral

\footnotetext{
University of South Africa, 2002, pp. 452-453. Lire aussi Esambo Kangashe J.-L., « Le Président Mobutu et le Premier ministre sous l'Acte constitutionnel de la transition : duel ou duo ? », Afrika Focus, vol. 14, n 2, 1998, pp. 161-178.

${ }^{52}$ Lire l'art. 1 point 21 de la Constitution Gabonaise de 1991 telle que révisée actuellement.

${ }^{53}$ Art. 93 al. 4 de la loi organique portant organisation et fonctionnement de la Cour constitutionnelle, in J.O/RDC, Numéro spécial d'octobre 2013.

${ }^{54}$ Art. 87 de la loi organique sur la Cour constitutionnelle au Gabon.
} 
soutenue par le peuple, peut se servir en RD Congo (rectification d'erreur matérielle) et au Gabon (procédure en révision) pour pouvoir amener le juge électoral à revoir sa décision.

\section{Conclusion}

Nous avons essayé dans cette étude, de décrire le rôle partisan et dangereux que joue le juge électoral dans le règlement des litiges relatifs au contentieux électoral de l'élection présidentielle et législative en Afrique francophone et particulièrement, au Gabon et en RD Congo. Le regard a été fixé sur le contentieux de l'élection présidentielle et législative de 2016 au Gabon et celles de 2011 et 2018 en RD Congo. L'économie générale du droit électoral dans les deux pays étudiés, reconnaît la compétence de la Cour constitutionnelle en matière de contentieux de l'élection présidentielle et législative nationale. Mais le travail de ces Cours constitutionnelles en matière des contentieux électoraux de l'élection présidentielle et législative, dénote véritablement un rendez-vous manqué dans un État de droit, le constat restant mitigé par rapport à l'apport du juge électoral dans la légitimation du processus électoral et des résultats finaux.

Ce qui fait que les arrêts de deux Cours évoquées, n’ont fait qu'amplifier les violences post-électorales au côté des discours politiques animés par des contestations et la manipulation populaire, qui ont donné lieu, à des violations massives des droits de l'homme à travers une répression sanglante des manifestants, et à l'entérinement des push électoraux au Gabon et en RDC. L'indépendance du juge électoral, reste la seule garantie pour la sauvegarde de la souveraineté du peuple au Gabon et en RD Congo. Une réforme sur le mode de désignation des animateurs de la Cour constitutionnelle, serait un gage pour aboutir à une justice électorale et constitutionnelle indépendante clé de voute de l'État de droit dans une démocratie. Cette réforme viendrait nécessairement d'une simple volonté politique des acteurs, ayant pour idéal de pouvoir placer l'État de droit au-dessus des enjeux politiques d'accession ou de conservation du pouvoir.

\section{Bibliographie}

\section{Textes juridiques}

- Constitution congolaise du 18 février 2006, telle que révisée en 2011, in J.O/RDC, $47^{\text {ème }}$ année, $n^{\circ}$ spécial du 18 février 2006.

- Constitution du Gabon de 1991 révisé à ce jour.

- $\quad$ Loi électorale de 2017 en RD Congo. 
- 93 al. 4 de la loi organique portant organisation et fonctionnement de la Cour constitutionnelle, in J.O/RDC, Numéro spécial d'octobre 2013.

\section{Jurisprudence}

- CC/Gabon, Décision n50/CC du 23 septembre 2016 rendue en reformation des résultats de l'élection présidentielle.

- CSJ, Arrêt R.E 007 du 28 décembre 2011.

- CJS, Arrêt R.C.E. PR.009 du 27 novembre 2006 et l'arrêt R.E. 007.

- $\quad$ CC, RC 001/PR du 19 janvier 2019.

\section{Doctrine}

- CENTRE CARTER, Obligations et normes électorales : manuel d'évaluation, CENTRE CARTER, Atlanta, 2014.

- COMMISSION DE VENISE, Le droit électoral, Éditions du Conseil de l'Europe, Strasbourg, 2000.

- CHEVALLIER Jacques, «Droit constitutionnel et institutions politiques : les mésaventures d'un couple fusionnel », in HAL, Mélanges en l'honneur de Pierre Avril, Montchrestien, Paris, 2000.

- DE GAUDUSSON Jean du Bois, «Les élections à l'épreuve de l'Afrique », in Cahiers du Conseil Constitutionnel nº 13 (dossier : la sincérité du scrutin) - janvier 2003.

- ESAMBOKANGASHE J.-L., « Le Président Mobutu et le Premier ministre sous l'Acte constitutionnel de la transition : duel ou duo ? », Afrika Focus, vol. 14, n 2, 1998.

- El Hadj MBODJ, « Les garanties et éventuels statuts de l'opposition en Afrique » in mandat, rôles et fonctions des pouvoirs constitués dans le nouveau système politique de la République démocratique du Congo, modules de formation du PNUD, Kinshasa, 2007.

- ISSAKA SOUARE, Les partis politiques de l'opposition en Afrique de l'Ouest et leur quête pour le pouvoir d'État : cas du Guinée, Ghana, Benin, Thèse de doctorat en science politique, Université du Québec à Montréal, 2010.

- FAll A., "Le processus de démocratisation en Afrique francophone : le juge de l'élection dans l'impasse ?" (Essai de prospective), in Démocratie et élections dans l'espace francophone, Bruxelles, Bruylant, 2010.

- MINANI BUHUZO R, «République Démocratique du Congo : La démocratie à l'épreuve de la géopolitique et la bonne gouvernance », Congo-Afrique, n 423, mars 2008. 
- Koкоroкo D., "Les élections disputées : réussites et échec", Pouvoir, Revue d'étude constitutionnelle et politique, $\mathrm{n}^{\mathrm{0}}$ 129, Avril 2009.

- MBATA André, «Arrêt de la Cour constitutionnelle : nomination des commissaires spéciaux, respect de la Constitution, l'élection présidentielle, de la Cour constitutionnelle et de l'article 64 », in Social Science Research Council, New-York, 28 avril 2016.

- MBATA André, The Road to Constitutionnalism and Democracy in Post-colonial Africa. The Case of Democratic Republic of Congo, Doctoral Thesis, University of South Africa, 2002.

- NTWALI Valéry, Le Rôle de l'opposition politique dans une démocratie représentative : le système multipartiste congolais, Ed. Universitaires Européennes, Berlin, 2015.

- SENE M, « Le juge constitutionnel face au défi de la continuité démocratique en Afrique noire francophone ", in Presses de l'Université de Toulouse 1 Capitole, 2014.

\section{Adresses électroniques}

- https://le-politiste.com/introduction-au-droit-electoral/

- $\quad$ https://www.france24.com/fr/20160908-gabon-mediation-union-africaine-reporteesine-die-nouvel-ordre-ping-bongo

- https://fr.africanews.com/2019/01/14/contentieux-electoral-en-rdc-la-courconstitutionnelle-examine-la-requete-de//

- $\quad$ http://www.rfi.fr/afrique/20190118-elections-rdc-rapport-detaille-cenco-resultats-ceni. 
Plan de l'article

Introduction 2

I. L'application du droit électoral par le juge constitutionnel au Gabon et en RD Congo......... 5

A. La compétence électorale reconnue au juge constitutionnel .......................................5

B. Les éléments mettant à mal les actions du juge constitutionnel en matière électoral au Gabon et en RD Congo.....

a) Les préalables à la tenue d'une élection (enrôlement des électeurs et mode de scrutin ou son changement)

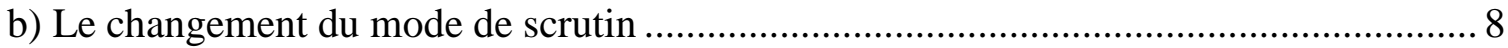

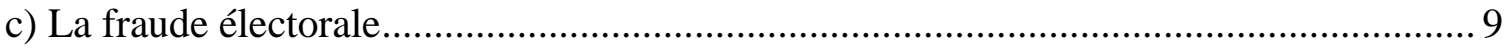

d) La nature même du contentieux électoral .................................................................... 10

e) La modalité de désignation des juges constitutionnels ............................................. 11

II. L'interprétation laconique du droit électoral par le juge constitutionnel au Gabon et en RD Congo : gage des crises post-électorales ................................................................... 11

A. Tendances jurisprudentielles du juge électoral au Gabon et en RD Congo....................... 12

1. Le juge électoral gabonais face à la requête de Jean Ping du 8 septembre 2016 ............. 12

a) Contenu de la requête de Jean Ping ...................................................................... 12

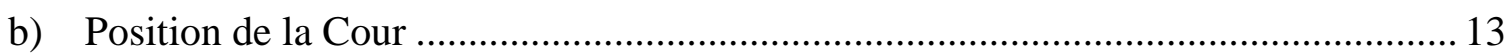

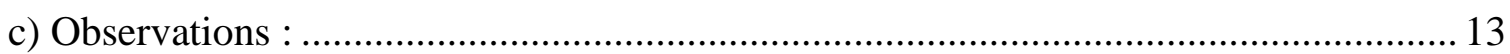

2. Le juge électoral congolais face au contentieux de 2011 et 2018 ................................... 15

a) Contenu de la requête de l'Union pour la nation congolaise (UNC) de Vital Kamerhe 15

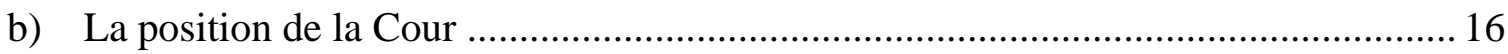

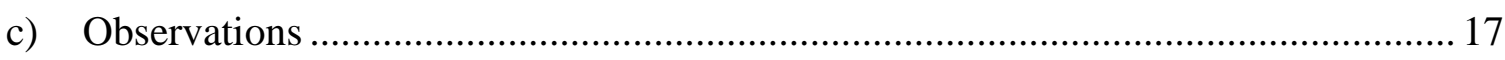

3. Contenu de la requête de Fayulu candidat de Lamuka ................................................. 18

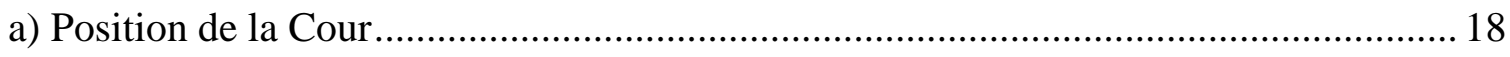

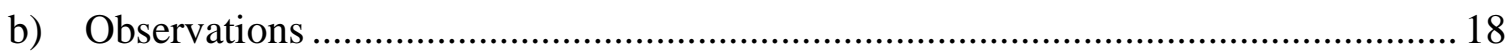

B. Le mécanisme constitutionnel du rétablissement de la vérité des urnes.......................... 19

1. Le rétablissement de la vérité des urnes par la voie révolutionnaire ...............................20

2. La rectification d'erreur matérielle (RDC) et procédure en révision (Gabon) .................. 22

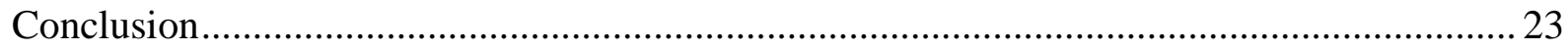

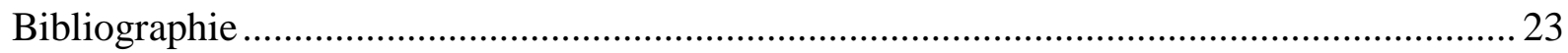

